\title{
Targeted enzyme prodrug therapy for metastatic prostate cancer - a comparative study of L-methioninase, purine nucleoside phosphorylase, and cytosine deaminase
}

\author{
Katrin P Guillen ${ }^{1}$, Carla Kurkjian ${ }^{2,3}$ and Roger G Harrison ${ }^{1,3^{*}}$
}

\begin{abstract}
Background: Enzyme prodrug therapy shows promise for the treatment of solid tumors, but current approaches lack effective/safe delivery strategies. To address this, we previously developed three enzyme-containing fusion proteins targeted via annexin $\vee$ to phosphatidylserine exposed on the tumor vasculature and tumor cells, using the enzymes L-methioninase, purine nucleoside phosphorylase, or cytosine deaminase. In enzyme prodrug therapy, the fusion protein is allowed to bind to the tumor before a nontoxic drug precursor, a prodrug, is introduced. Upon interaction of the prodrug with the bound enzyme, an anticancer compound is formed, but only in the direct vicinity of the tumor, thereby mitigating the risk of side effects while creating high intratumoral drug concentrations. The applicability of these enzyme prodrug systems to treating prostate cancer has remained unexplored. Additionally, target availability may increase with the addition of low dose docetaxel treatment to the enzyme prodrug treatment, but this effect has not been previously investigated. To this end, we examined the binding strength and the cytotoxic efficacy (with and without docetaxel treatment) of these enzyme prodrug systems on the human prostate cancer cell line PC-3.

Results: All three fusion proteins exhibited strong binding; dissociation constants were $0.572 \mathrm{nM}$ for L-methioninase-annexin $\mathrm{V}$ (MT-AV), $0.406 \mathrm{nM}$ for purine nucleoside phosphorylase-annexin $\mathrm{V}$ (PNP-AV), and $0.061 \mathrm{nM}$ for cytosine deaminase-annexin V (CD-AV). MT-AV produced up to 99\% cell death $(p<0.001)$ with limited cytotoxicity of the prodrug alone. PNP-AV with docetaxel created up to $78 \%$ cell death $(p<0.001)$ with no cytotoxicity of the prodrug alone. CD-AV with docetaxel displayed up to $60 \%$ cell death $(p<0.001)$ with no cytotoxicity of the prodrug alone. Docetaxel treatment created significant increases in cytotoxicity for PNP-AV and CD-AV.

Conclusions: Strong binding of fusion proteins to the prostate cancer cells and effective cell killing suggest that the enzyme prodrug systems with MT-AV and PNP-AV may be effective treatment options. Additionally, low-dose docetaxel treatment was found to increase the cytotoxic effect of the annexin V-targeted therapeutics for the PNP-AV and CD-AV systems.
\end{abstract}

Keywords: Enzyme prodrug therapy, Vascular-targeted, Docetaxel, Annexin V, Prostate cancer

\footnotetext{
* Correspondence: rharrison@ou.edu

'Bioengineering Center and the School of Chemical, Biological and Materials Engineering, University of Oklahoma, Norman, OK, USA

${ }^{3}$ Stephenson Cancer Center, Health Sciences Center, University of Oklahoma,

Oklahoma City, OK, USA

Full list of author information is available at the end of the article
} 


\section{Background}

Prostate cancer (PC) is the most common non-skin malignancy and the second leading cause of cancer-related death in American men [1], yet remains essentially incurable. Since the introduction of PSA specific screening, the lethality of prostate cancer stems not from a lack of early detection but more commonly from the failure of locoregional therapies creating a need for improved systemic therapies [2]. Currently, most single-agent anticancer drugs face challenges due to increased multidrug resistance [3], pharmacokinetic limitations [4,5], and restricted clinical dosage or frequency of administration due to cytotoxicity in non-cancerous tissues [6-8].

Antibody-directed enzyme prodrug therapy (ADEPT), gene-directed enzyme prodrug therapy (GDEPT), and viral-directed enzyme prodrug therapy (VDEPT) have been investigated as means to utilize enzymes to convert relatively non-toxic prodrugs into clinically relevant concentrations of cytotoxic drugs directly at tumor sites. However, all three of these approaches have significant limitations [9-11]. To improve upon the clinical applicability, efficacy, and safety of enzyme prodrug therapy, we previously developed three fusion proteins (FPs), each targeted to primary tumors, their metastases, and the tumor vasculature. This dual targeting strategy allows for two distinct mechanisms of killing: (i) via the direct action of the cytotoxic drug on the tumor cells, and (ii) by killing tumor vasculature endothelial cells and thereby effectively cutting off the tumor blood supply. Vascular targeting makes these FPs an attractive option because endothelial cells are relatively genetically-stable, easily-accessible targets that enable therapeutic effect amplification through tumor infarction, as well as tumor-type independent targeting [12]. PC is especially well suited to this dual targeting strategy as prostate carcinomas have been shown to have approximately twice the vascular density of healthy prostate tissue [13] and microvessel density serves as a predictor of cancer-specific survival [14]. To date, the efficacy of these targeted enzyme prodrug systems on PC has remained unexplored.

Human annexin V (AV) is used to target each FP. AV has a strong affinity to the anionic phospholipid phosphatidylserine (PS), normally tightly segregated to the inner leaflet in eukaryotic plasma membranes [15], but robustly and consistently expressed on the outer leaflet in a wide range of cancer cell lines, their metastases [16,17], and the luminal side of tumor endothelium $[18,19]$. To maximize FP binding to tumor cells, we investigated treatment with docetaxel, a tubulin/microtubule targeting chemotherapeutic agent [20], which is becoming increasingly important in combination therapies for metastatic, hormone-refractory PC [21]. Therapeutic docetaxel dosage is limited by drug toxicity [7] but a single subtoxic dose has been shown to increase PS exposure on tumor endothelium by $\sim 70 \%$ without causing apoptosis or changing PS exposure on normal endothelium [22]. This large increase in AV binding sites has the potential to increase the cytotoxic power of our enzyme prodrug systems.

We previously developed three AV-targeted FPs, each containing a non-human enzyme [23-25]. The enzymes utilized are:

(i) L-methioninase (MT), which converts L-selenomethionine (SeMet) to toxic methylselenol, $\alpha$-ketobutyrate, and ammonia [26]. MT also converts the amino acid methionine to methanethiol, which provides a second point of attack since most cancer cells exhibit increased methionine-dependence $[27,28]$. PC cell lines have shown sensitivity to non-targeted MT/SeMet treatment in vitro [29].

(ii) Purine nucleoside phosphorylase (PNP), which converts fludarabine (FD) into highly cytotoxic 2-fluoroadenine (2-FA) that incorporates into DNA/RNA, thereby effectively killing both dividing and nondividing cells [30]. PNP exhibits a powerful bystander effect [31,32], and PC cells have shown sensitivity to PNP/FD GDEPT treatment [33-35].

(iii) Cytosine deaminase (CD), which converts the nucleoside analog 5-fluorocytosine (5-FC) to the more toxic pyrimidine analog 5-fluorouracil (5-FU), metabolites of which misincorporate into DNA/ RNA and inhibit the nucleotide synthesis enzyme thymidylate synthetase [36]. PC cell lines have shown sensitivity to 5 -FU and GDEPT CD/5-FC treatment [37].

To address the vascular targeting capabilities of these enzyme prodrug systems, we have previously shown that all three FPs bind tightly to PS expressing human abdominal aorta endothelial cells (HAAE-1) in vitro, with dissociation constants ranging from 0.5-1.5 nM [23-25]. Cytotoxic efficacy of our FP systems on HAAE-1 cells has also been demonstrated previously in vitro, with cell killing ranging from 5-100\% [23-25]. We have validated these in vitro methods for determining vascular targeting/cytotoxic efficacy via the successful transition of the MT-AV/ SeMet system in vivo for mice with implanted MDA-MB231 breast tumors [38].

In the present study, we characterize the binding and evaluate the in vitro anticancer efficacy of three enzyme prodrug systems on $\mathrm{PC}-3$ human prostate carcinoma cells in the presence and absence of low-dose docetaxel treatment.

\section{Methods}

\section{Expression and purification of fusion proteins}

All FPs were expressed and purified as described previously [23-25]. Briefly, polymerase chain reaction (PCR) 
was used amplify genes encoding each enzyme, a six residue flexible linker, annexin $\mathrm{V}$, an $\mathrm{N}$ - or $\mathrm{C}$ - terminal $\mathrm{His}_{6}$ tag, and an engineered HRV $3 \mathrm{C}$ protease cleavage site. Plasmids containing each FP were created via transformation of NovaBlue competent cells and then expressed in E.coli BL21 (DE3) cells. Recombinant FPs were produced and purified according to the procedure of Zang et al. [39] using immobilized metal $\left(\mathrm{Ni}^{2+}\right)$ affinity chromatography. $\mathrm{The} \mathrm{His}_{6}$ tag was removed during purification by cleavage with HRV-3C protease (Merck, Darmstadt, Germany). FPs were lyophilized and stored at $-80^{\circ} \mathrm{C}$.

\section{Cell culture}

The PC-3 human prostate adenocarcinoma cell line was obtained from the American Type Culture Collection (ATTC, Manassas, VA, USA) and cultured in F-12 K medium (ATTC) supplemented with 10\% fetal bovine serum, $100 \mathrm{U} / \mathrm{ml}$ penicillin, and $100 \mu \mathrm{g} / \mathrm{ml}$ streptomycin (all from Atlanta Biologics, Flowery Branch, GA, USA) at $37^{\circ} \mathrm{C}$ in a $5 \% \mathrm{CO}_{2}$ atmosphere. Cells were passaged at 70-80\% confluence, 2-3 times per week, less than 12 times during the course of experiments.

\section{In vitro binding assays}

Cells were grown in T-75 flasks to $70-80 \%$ confluence, plated at $50 \mathrm{k}$ cells/well in 24-well cell culture plates, and allowed to grow to $90 \%$ confluence. Dissociation constants were determined as described previously [23-25]. Briefly, cells were fixed with $0.25 \%$ glutaraldehyde in PBS, then quenched with $50 \mathrm{nM} \mathrm{NH}_{4} \mathrm{Cl}$ in PBS. After a $1 \mathrm{~h}$ of incubation with $0.5 \%$ BSA in PBS, cells were washed, and varying concentrations $(0-20 \mathrm{nM})$ of SureLINK biotin (KPL, Gaithersburg, MD, USA) labeled FPs were added and allowed to bind at $37^{\circ} \mathrm{C}$ for $2 \mathrm{~h}$. Cells were washed with PBS containing 0.5\% BSA and treated with streptavidinhorseradish peroxidase $(2 \mu \mathrm{g} / \mathrm{ml}, \mathrm{KPL})$ for $1 \mathrm{~h}$ at room temperature. Cells were washed, and HRP was quantified via chromogenic substrate $o$-phenylenediamine $(0.4 \mathrm{mg} / \mathrm{ml})$ in $0.05 \mathrm{mM}$ phosphate-citrate buffer $(\mathrm{pH} 5.0)$ containing $0.012 \%$ hydrogen peroxide. Since $\mathrm{Ca}^{2+}$ is essential for AV binding to PS, the above procedure was conducted in the presence of $2 \mathrm{mM} \mathrm{Ca}^{2+}$ (total binding) and in the absence of $\mathrm{Ca}^{2+}$ with $5 \mathrm{mM}$ EDTA to chelate any residual $\mathrm{Ca}^{2+}$ (non-specific binding). All experiments contained a blank subjected to the same procedure but with $0 \mathrm{nM} \mathrm{FP}$.

\section{In vitro enzyme prodrug cytotoxic efficacy}

Studies were carried out over a 6-day (MT-AV, PNP-AV) or 9-day $(\mathrm{CD}-\mathrm{AV})$ treatment cycle. Cells were plated as described previously, but only allowed to reach 50-60\% confluence. Prior to the first viability assay, selected wells were pre-treated with $50 \mathrm{pM}$ docetaxel (Biotang, Waltham, MA, USA). All medium was enhanced with $2 \mathrm{mM} \mathrm{Ca}^{2+}$ since annexin $\mathrm{V}$ binding is calcium-dependent. Medium for
MT-AV cytotoxicity studies was also supplemented with $0.02 \mathrm{mM}$ pyridoxal phosphate (co-factor). Cells were treated with a saturating concentration of FP (100 nM) every 3 days for $2 \mathrm{~h}$ at $37^{\circ} \mathrm{C}$ in accordance with previous binding stability studies [23-25]. Each day, medium was replaced with medium containing varying concentrations of prodrug (L-SeMet and 5-FC from Fisher Scientific, Waltham, MA, USA and FD from VWR, Radnor, PA, USA) or drug analog (2-FA from Fisher Scientific and 5-FU from Sigma-Aldrich, St. Louis, MO, USA) with or without 50 pM docetaxel. This docetaxel concentration was chosen since it is similar to the level previously reported to lead to PS exposure in vitro without a cytotoxic effect on cells [22]. An Alamar Blue (Invitrogen, Grand Island, NY, USA) assay was preformed every 2-3 days to measure cell viability [40]. Cells were incubated with 10\% Alamar Blue in fresh media for $4 \mathrm{~h}$ at $37^{\circ} \mathrm{C}$. From each well, $250 \mu \mathrm{l}$ was transferred to an opaque 96-well plate, and fluorescence (530/ $590 \mathrm{~nm}$ ) was read on a microtiter plate reader. Cells were washed twice after each Alamar Blue assay and three times after each FP incubation before prodrug/drug treatments were added.

\section{Data analysis}

All treatments were run in triplicate. Dissociation constants were obtained using Prism 5 software (GraphPad, La Jolla, CA, USA). Statistical significance was determined with Prism 5 via a one-way ANOVA employing the Tukey-Kramer multiple comparisons test.

\section{Results}

\section{Binding strength}

The ability of each FP to bind to PS on the PC-3 cell surface was determined by measuring the total binding and non-specific binding and subtracting to obtain specific binding, with typical results shown in Figure 1 for MTAV. The dissociation constant $\left(K_{d}\right)$ was calculated utilizing a one-site, non-competitive binding model, and $K_{d}$ values are presented in Table 1.

\section{Enzyme prodrug cytotoxic efficacy}

We evaluated the cytotoxic effect of each enzyme prodrug therapy on PC-3 cells by comparing the cell viability on days 2, 4, and 6 (MT-AV and PNP-AV) or days 3, 6, and 9 (CD-AV) to day 0 on a per well basis, and results are presented as percent viability compared to day 0 . Statistical significance was established by comparing cells treated with varying concentrations of prodrug (or drug analog, if available) to their corresponding control groups treated with $0 \mu \mathrm{M}$ drug/prodrug on the same day (\#, $p<0.05 ;{ }^{*}, p<0.01$; and $\left.{ }^{* *}, p<0.001\right)$. Additionally, cells treated with $50 \mathrm{pm}$ docetaxel were compared to cells not treated with docetaxel at the same concentrations of prodrug/drug on the same day $(\wedge, p<0.05 ;+, p<0.01$; and,$++ p<0.001)$. 


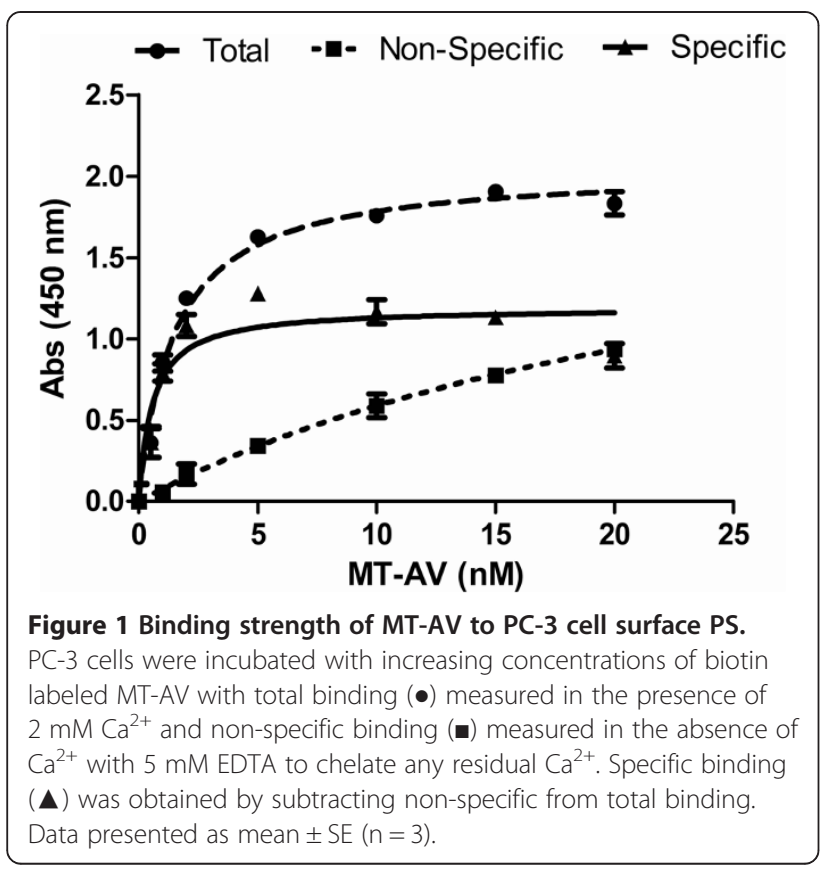

The cytotoxic effect of SeMet conversion by MT-AV was evaluated over 6 days with SeMet concentrations ranging from 0 to $1000 \mu \mathrm{M}$ with $50 \mathrm{pM}$ docetaxel (data not shown) and without docetaxel (Figure 2). MT-AV/ SeMet treatment caused significant cytotoxicity starting at $250 \mu \mathrm{M}$ SeMet, resulting in $64 \%$ viability by day 2 and $14 \%$ viability by day 6 , with no growth inhibition for SeMet alone. At SeMet concentrations above $250 \mu \mathrm{M}, \mathrm{MT}-\mathrm{AV} /$ SeMet killing velocity increased and near complete killing was achieved by day 6 , with only slight growth inhibitory effects of SeMet alone. The addition of docetaxel treatment created no significant additional decreases in cell viability.

The cytotoxic effect of 2-FA converted from FD by PNP-AV in the presence (Figure 3(a)) and absence (see Additional file 1: Figure S1) of docetaxel treatment was determined over 6 days with FD or 2-FA concentrations ranging from 0 to $10 \mu \mathrm{M}$. PNP-AV in combination with $5 \mu \mathrm{M}$ FD was the lowest concentration of prodrug that showed significant cytotoxic effects, reaching $37 \%$ viability by day 6 with docetaxel treatment and $50 \%$ viability without docetaxel treatment. Cytotoxicity effects increased with increasing FD concentration up to $10 \mu \mathrm{M}$, reaching $22 \%$ viability by day 6 with docetaxel treatment and 37\% viability without docetaxel treatment. Treatment with

Table 1 Dissociation constant $\left(K_{d}\right)$ of each fusion protein binding to PC-3 cells

\begin{tabular}{ll}
\hline Fusion protein & $\mathbf{K}_{\mathbf{d}} \pm \mathbf{S E}(\mathbf{n M})$ \\
\hline MT-AV & $0.572 \pm 0.281$ \\
PNP-AV & $0.406 \pm 0.108$ \\
CD-AV & $0.061 \pm 0.026$ \\
\hline
\end{tabular}

PNP-AV/FD was statistically indistinguishable from 2-FA treatment alone at concentrations $\geq 5 \mu \mathrm{M}$ for docetaxel treated cells, but for non-docetaxel treated cells this treatment similarity did not occur at the concentrations evaluated (see Additional file 1: Figure S1). FD treatment alone did not show any cytotoxic effects at concentrations $\leq$ $10 \mu \mathrm{M}$. Treatment only with docetaxel did not affect PC-3 cells, but the addition of docetaxel significantly enhanced PNP-AV/FD cytotoxic efficacy at $5 \mu \mathrm{M}$ FD concentrations and above as indicated $(\wedge,+,++)$ in Figure 3(a). Additional decreases in $\%$ cell viability that occurred with docetaxel treatment are presented in Figure 3(b) for FD concentrations of $5,7.5$, and $10 \mu \mathrm{M}$. On day 2 , additional decreases in \% cell viability ranged from $15-19 \%$. The highest additional cytotoxicity occurred on day 4 (22-27\%), and the effect diminished by day 6 .

The cytotoxic effect of 5-FU converted from 5-FC by CD-AV with (Figure 4(a)) and without (see Additional file 1: Figure S2) docetaxel treatment was evaluated over 9 days with concentrations of 5-FC/5-FU ranging from 0 to $5000 \mu \mathrm{M}$. CD-AV/5-FC treatment caused significant cytotoxicity at all concentrations above $500 \mu \mathrm{M}$ but was most effective at $5000 \mu \mathrm{M} 5$-FC resulting in $40 \%$ viability by day 9 with docetaxel treatment and 44\% without docetaxel treatment. No significant increases in cytotoxicity occurred past $5000 \mu \mathrm{M}$ CD-AV/5-FC or 5 -FU treatment (data not shown). 5-FC treatment alone exhibited no cytotoxic effect for both docetaxel and nondocetaxel treated cells. Treatment with the drug analog 5 -FU showed significantly more cytotoxic effects than treatment with $\mathrm{CD}-\mathrm{AV} / 5-\mathrm{FC}$ from day 6 onwards and resulted in $\sim 6 \%$ viability for both docetaxel and non/docetaxel groups. Treatment only with docetaxel had no effect on PC-3 cells, but the addition of docetaxel significantly affected the killing efficacy of the CD-AV/5-FC system as indicated in Figure 4(a) $(\wedge,+)$. The additional decreases in $\%$ viability as a result of docetaxel addition are presented in Figure 4(b). Docetaxel affected CD-AV/ 5-FC efficacy in an inverse dose dependent manner, with respect to the prodrug, as the largest additional decreases in \% viability consistently occurred at $1000 \mu \mathrm{M}$ 5-FC and the smallest additional decreases were consistently seen at $5000 \mu \mathrm{M} 5$-FC. As for PNP-AV/FD, the impact of docetaxel was greatest in the middle of the study reaching an additional decrease in $\%$ viability of $26 \%$ on Day 6 for $1000 \mu \mathrm{M}$ 5-FC.

\section{Discussion}

The MT-AV/SeMet enzyme prodrug system emerged as a promising treatment option as it displayed significant cytotoxicity in vitro at feasible in vivo SeMet treatment concentrations. The median lethal dose $\left(\mathrm{LD}_{50}\right)$ of SeMet in vivo for female nude mice is $12.5 \mathrm{mg} / \mathrm{kg}$ [41,42], which translates to $\sim 1100 \mu \mathrm{M}$ in vitro. A high degree of 


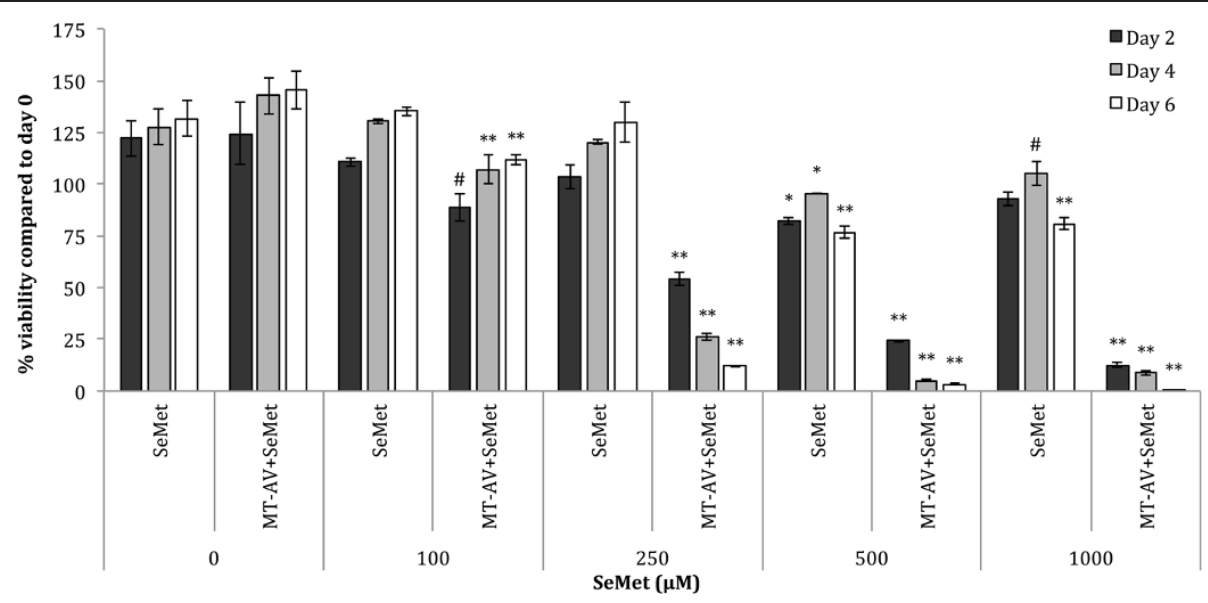

Figure 2 Effect of SeMet conversion by MT-AV on PC-3 cell viability. Cells treated with varying concentrations of SeMet were compared their corresponding control groups treated with $0 \mathrm{nM}$ concentrations on the same day, and significant differences are denoted by \# ( $p<0.05)$, * $(p<0.01)$, and $*^{*}(p<0.001)$. Data presented as mean \pm SE $(n=3)$.
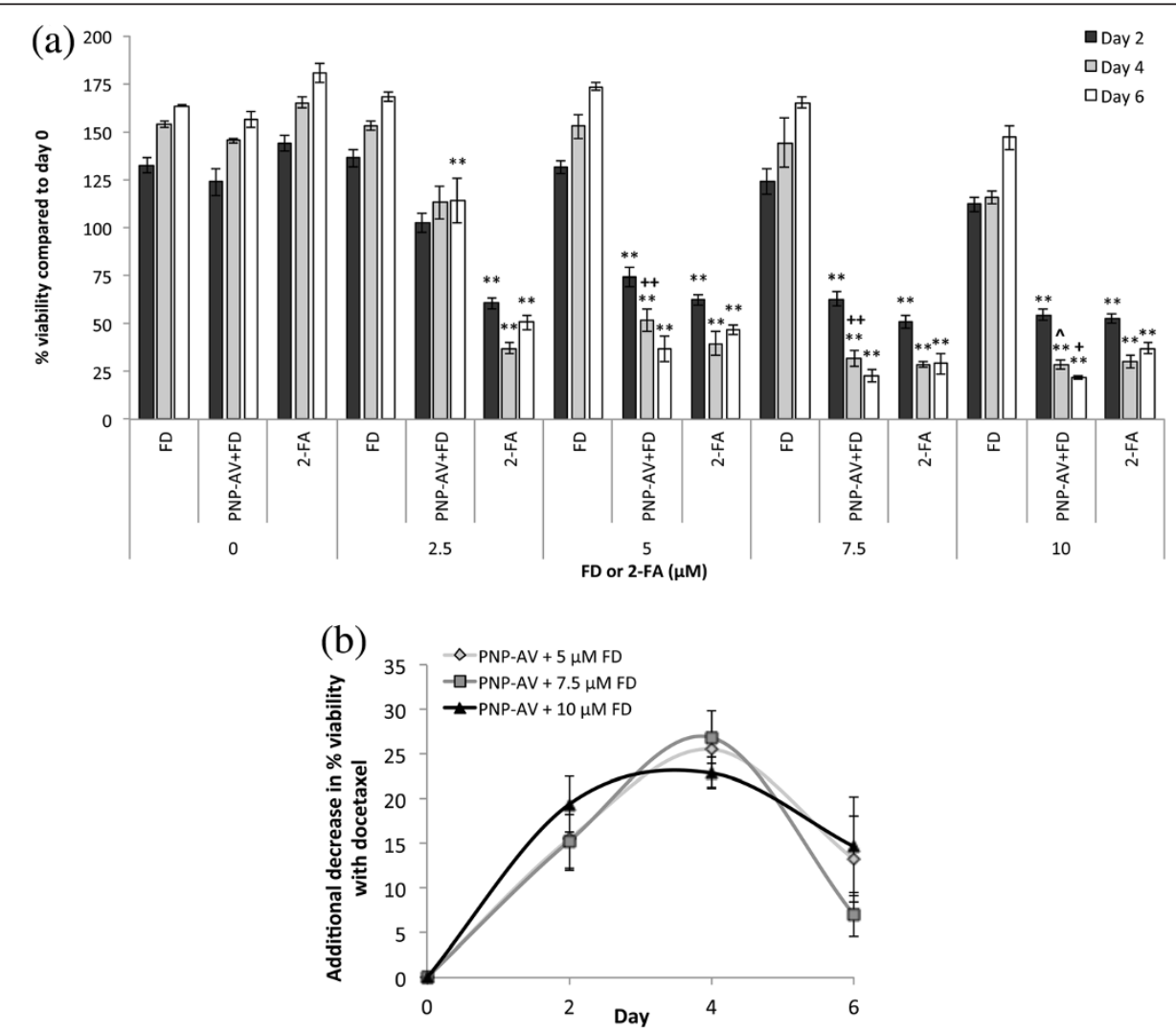

Figure 3 Effect of FD conversion by PNP-AV with 50 pM docetaxel on PC-3 cell viability. (a) Cells treated with varying concentrations of FD or 2-FA were compared to their corresponding control groups treated with $0 \mathrm{nM}$ concentrations on the same day, and significant differences are denoted by \# $(p<0.05),{ }^{*}(p<0.01)$, and ${ }^{* *}(p<<0.001)$. Cells treated with $50 \mathrm{pm}$ docetaxel (shown) were compared to cells not treated with docetaxel (see Additional file 1: Figure S1) at the same concentrations of FD or 2-FA on the same day, and significant differences are denoted by $\wedge(p<0.05),+(p<0.01)$, and $++(p<0.001)$. Data presented as mean $\pm \operatorname{SE}(n=3)$. (b) Additional decreases in cell viability afforded by the addition of $50 \mathrm{pM}$ docetaxel to the PNP-AV system efficacy, shown for prodrug titers for which docetaxel treatment influenced treatment outcome. Results shown as non-docetaxel treated \% viability minus docetaxel treated \% viability to obtain a measure of additional cell killing with docetaxel treatment that alone has no significant effect on cell growth. Data presented as mean $\pm S E(n=6)$. 

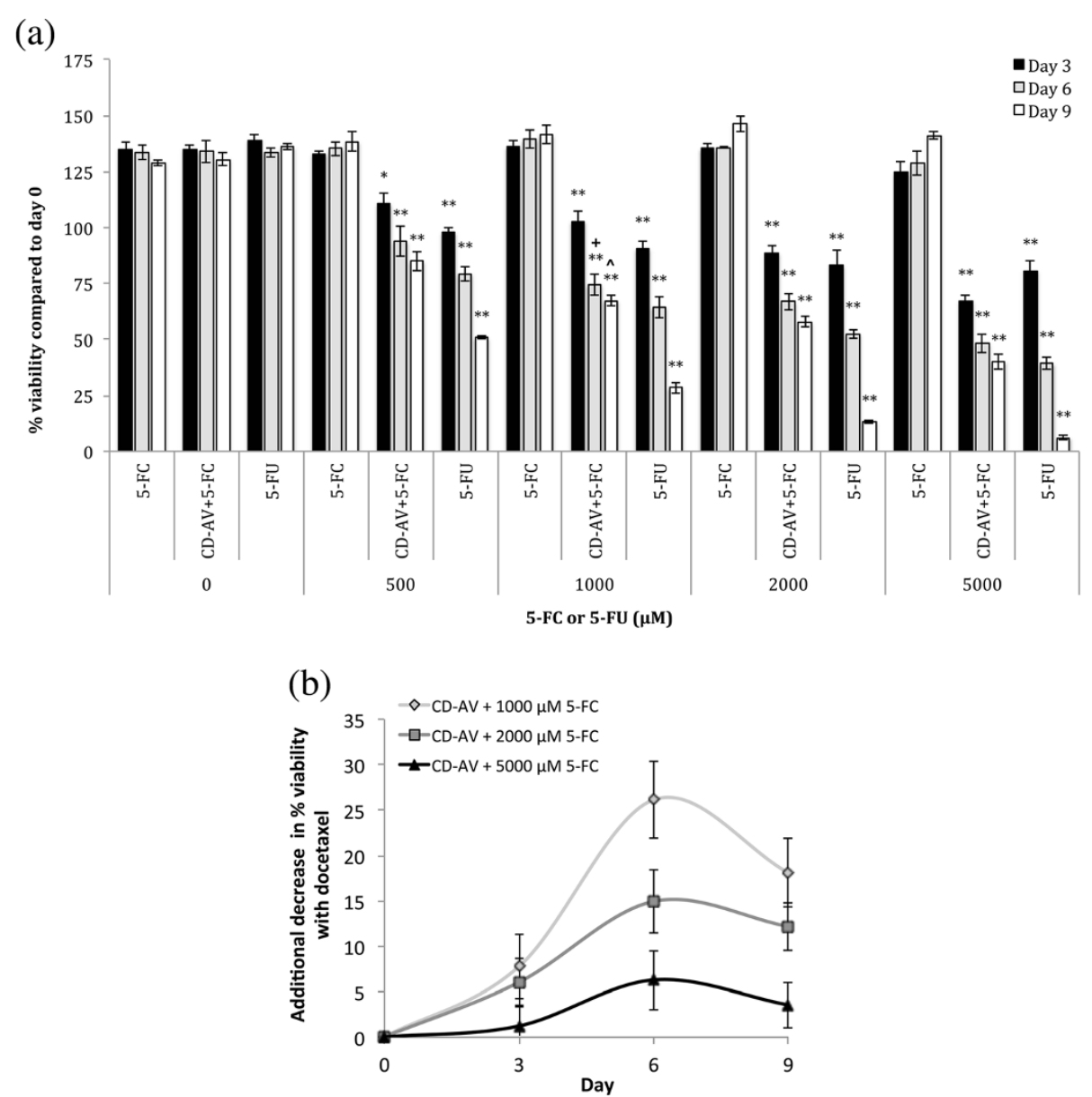

Figure 4 Effect of CD-AV conversion of 5-FC with 50 pM docetaxel treatment on PC-3 cell viability. (a) Cells treated with varying concentrations of 5-FC or 5-FU were compared their corresponding control groups treated with $0 \mathrm{nM}$ concentrations on the same day, and significant differences are denoted by \# $(p<0.05),{ }^{*}(p<0.01)$, and ${ }^{* *}(p<0.001)$. Cells treated with $50 \mathrm{pm}$ docetaxel were compared to cells not treated with docetaxel (see Additional file 1: Figure S2) at the same concentrations of 5-FC or 5-FU on the same day and significant differences are denoted by $\wedge(p<0.05)$ or $+(p<0.01)$. Data presented as mean \pm SE $(n=3)$. (b) Additional decreases in cell viability by the addition of $50 \mathrm{pM}$ docetaxel, which alone has no effect on cell viability, for prodrug concentrations where docetaxel additional affected treatment outcomes. Results shown as non-docetaxel treated \% viability minus docetaxel treated \% viability to obtain a measure of additional cell killing with docetaxel treatment that alone has no significant effect on cell growth. Data presented as mean \pm SE $(n=6)$.

prostate cancer cell killing was achieved with SeMet concentrations as low as $250 \mu \mathrm{M}$ with minimal cytotoxicity of SeMet alone, suggesting a feasible window of opportunity for in vivo treatment translation.

PNP-AV also emerged as a feasible option for treating $\mathrm{PC}$ as it displayed high killing velocity and killing efficacy, both of which are important for clinical translation. PNP$\mathrm{AV}$ also showed the most robust increase in cell killing efficacy in the presence of docetaxel. PNP-AV/FD (with docetaxel) created up to $78 \%$ cytotoxicity over 6 days at an FD concentration of $10 \mu \mathrm{M}$ in vitro, which translates to less than $0.1 \%$ of the $\mathrm{LD}_{50}(\sim 1200 \mathrm{mg} / \mathrm{kg})$ for FD in female nude mice, indicating that this therapy could be administered with minimal harm to healthy tissues.

$\mathrm{CD}-\mathrm{AV} / 5$-FC treatment was not as effective or as rapid as MT-AV/SeMet or PNP-AV/FD treatment, and we therefore conclude that $\mathrm{CD}-\mathrm{AV} / 5-\mathrm{FC}$ would most likely not be effective in vivo. Additionally, the prodrug concentration necessary to elicit a cytotoxic effect was significantly higher than for the MT-AV and PNP-AV systems, although even at the highest level of prodrug, there was no effect of the prodrug by itself.

All three FPs exhibited relatively strong binding to PS on PC-3 cells with dissociation constants less than previously reported dissociation constants for AV alone to PS $(2.7-15.5 \mathrm{nM})[43,44]$. The multimeric structure of each FP likely allows for multiple AV to PS bonds per FP, and we believe this contributes to the observed strong binding of FPs to PS.

Subtoxic docetaxel treatment significantly but selectively increased the cytotoxic efficacy of our enzyme prodrug systems, suggesting that at least two of our FP/prodrug 
combinations are sensitive to the extent of PS outer leaflet exposure. Tumor xenografts in murine models expose $35 \%$ of PS on the external leaflet [18] (with $>10^{6}$ PS molecules per cell [22]), but docetaxel treatment can increase PS expose and thereby the number of potential FP binging sites by up to 70\% [22], effectively doubling the FP targets and thereby creating quicker and more powerful treatment. The additional decrease in cell viability caused by introducing docetaxel to the PNP-AV and CD-AV enzyme prodrug treatments is seen in Figures 3(b) and 4(b), respectively. The maximum effect was present at about the midpoint of each study, i.e. at day 4 in the PNP-AV system and at day 6 in the CD-AV system. We believe that the peak in this effect is a result of increased initial prodrug to drug turnover enabled by the increased presence of bound FP's due to the additional availability of PS binding sites. Therefore, the addition of docetaxel causes the enzyme prodrug treatment to speed up initially; and later as the number of viable cells dwindles, the effect becomes relatively less noticeable.

We employed subtoxic treatment levels of docetaxel, as we were interested in the PS exposure effects of docetaxel and not its cytotoxic capabilities. Not only did docetaxel treatment alone have no growth inhibitory or cytotoxic effects, but the addition of docetaxel treatment did not alter the cytotoxic efficacy of the drug analogs, 2-FA and 5-FU. This indicates there was no synergism present between the drugs generated by our enzyme prodrug therapies and docetaxel. Therefore, it is probable that the increased cytotoxic effect afforded by docetaxel treatment was in fact due to an increase in PS exposure providing an increase in available binding sites for our FPs.

Unexpectedly, docetaxel treatment did not increase MTAV cytotoxicity on PC-3 cells. We propose that this effect did not occur because the killing efficacy of the MT-AV system may already be saturated at feasible SeMet concentrations without docetaxel. Saturation could arise if the amount of MT-AV able to bind without docetaxel treatment is sufficient to convert the available SeMet, as any additional MT-AV binding would increase the initial SeMet turnover rate but would not ultimately affect the quantity of reactive oxygen species the cells are exposed to.

Further validation of the MT-AV and PNP-AV systems will consist of in vivo work in murine xenograft models. The immunogenicity of the FP systems can be addressed via functionalization of human homologs $[45,46]$ or via PEGylation [47].

\section{Conclusions}

In conclusion, we have substantiated the feasibility of two, novel, non-invasive treatments for prostate cancer and its metastases with minimal threat to healthy tissues. We were able to achieve both tight binding, with dissociation constants in the low nanomolar range, and excellent cytotoxic efficacy for the MT-AV and PNP-AV enzyme prodrug systems. Additionally we have shown the utility of subtoxic docetaxel treatment for increasing the cytotoxic potential of annexin V-targeted enzyme prodrug systems.

\section{Additional file}

Additional file 1: Figure S1. Effect of FD conversion by PNP-AV on PC-3 cell viability. Figure S2. Effect of 5-FC conversion by CD-AV on PC-3 cell viability.

\section{Abbreviations}

FP: Fusion protein; AV: Annexin V; MT: L-methioninase; PNP: Purine nucleoside phosphorylase; CD: Cytosine deaminase; PC: Prostate cancer; ADPET: Antibody-directed enzyme prodrug therapy; GDEPT: Gene-directed enzyme prodrug therapy; VDEPT: Viral-directed enzyme prodrug therapy; PS: Phosphatidylserine; FD: Fludarabine; 5-FC: 5-fluorocytosine; SeMet: L-selenomethionine; HAAE-1: Human abdominal aorta endothelial cells; $K_{d}$ : Dissociation constant; $L_{50}$ : Median lethal dose.

\section{Competing interests}

The only potential competing interest we declare is an application for a U.S patent on this targeted enzyme prodrug therapy system.

\section{Authors' contributions}

KPG, CK, and RGH designed the experiments. KPG conducted experiments and analyzed the data. KPG and RGH wrote the manuscript. All authors read and approved the final manuscript.

\section{Acknowledgements}

This research was supported by the University of Oklahoma Bioengineering Center.

\section{Author details}

${ }^{1}$ Bioengineering Center and the School of Chemical, Biological and Materials Engineering, University of Oklahoma, Norman, OK, USA.

${ }^{2}$ Oncology-Hematology Section, Health Sciences Center, University of Oklahoma, Oklahoma City, OK, USA. ${ }^{3}$ Stephenson Cancer Center, Health Sciences Center, University of Oklahoma, Oklahoma City, OK, USA.

Received: 2 April 2014 Accepted: 8 July 2014

Published: 22 July 2014

\section{References}

1. Siegel R, Naishadham D, Jemal A: Cancer statistics, 2012. CA Cancer J Clin 2012, 62:10-29.

2. Freytag $\mathrm{SO}$, Stricker $\mathrm{H}$, Movsas B, Kim JH: Prostate cancer gene therapy clinical trials. Mol Ther 2007, 15:1042-1052.

3. Gottesman MM, Fojo T, Bates SE: Multidrug resistance in cancer: role of ATP-dependent transporters. Nat Rev Cancer 2002, 2:48-58.

4. Hersh MR, Kuhn JG, Phillips JL, Clark G, Ludden TM, Hoff DD: Pharmacokinetic study of fludarabine phosphate (NSC 312887). Cancer Chemother Pharmacol 1986, 17:277-280.

5. Meikle SR, Matthews JC, Brock CS, Wells P, Harte RJA, Cunningham VJ, Jones T, Price P: Pharmacokinetic assessment of novel anti-cancer drugs using spectral analysis and positron emission tomography: a feasibility study. Cancer Chemother Pharmacol 1998, 42:183-193.

6. Cheung WY, Fralick R, Cheng S: The confused cancer patient: a case of 5-fluorouracil-induced encephalopathy. Curr Oncol 2008, 15:234-236.

7. Bissery MC, Nohynek G, Sanderink GJ, Lavelle F: Docetaxel (Taxotere): a review of preclinical and clinical experience. Part I: preclinical experience. Anticancer Drugs 1995, 6:339-355.

8. Matthews EJ, Kruhlak NL, Benz RD, Contrera JF: Assessment of the health effects of chemicals in humans: I. QSAR estimation of the maximum recommended therapeutic dose (MRTD) and no effect level (NOEL) of 
organic chemicals based on clinical trial data1. Curr Drug Discov Technol 2004, 1:61-76.

9. Del Vecchio S, Reynolds JC, Carrasquillo JA, Blasberg RG, Neumann RD Lotze MT, Bryant GJ, Farkas RJ, Larson SM: Local distribution and concentration of intravenously injected 1311-9.2. 27 monoclonal antibody in human malignant melanoma. Cancer Res 1989, 49:2783.

10. Singh $Y$, Palombo M, Sinko PJ: Recent trends in targeted anticancer prodrug and conjugate design. Curr Med Chem 2008, 15:1802-1826.

11. Ekblad M, Halldén G: Adenovirus-based therapy for prostate cancer. Curr Opin Mol Ther 2010, 12:421-431.

12. Hunt MA, Dachs GU, Currie MJ: Vascular Targeted Gene Therapy in Cancer Treatment. In New Gene Ther Cancer Res. Edited by Gustafsson WB. New York, NY, USA: Nova Science Publishers, Inc; 2008.

13. Bigler SA, Deering RE, Brawer MK: Comparison of microscopic vascularity in benign and malignant prostate tissue. Hum Pathol 1993, 24:220-226.

14. Franck Lissbrant I, Stattin P, Damber J-E, Bergh A: Vascular density is a predictor of cancer-specific survival in prostatic carcinoma. Prostate 1997, 33:38-45.

15. Bevers $E$, Comfurius $P, Z$ waal R: Regulatory mechanisms in maintenance and modulation of transmembrane lipid asymmetry: pathophysiological implications. Lupus 1996, 5:480-487.

16. Riedl S, Rinner B, Asslaber M, Schaider H, Walzer S, Novak A, Lohner K, Zweytick D: In search of a novel target-Phosphatidylserine exposed by non-apoptotic tumor cells and metastases of malignancies with poor treatment efficacy. Biochim Biophys Acta BBA-Biomembr 1808, 2011:2638-2645.

17. Utsugi T, Schroit AJ, Connor J, Bucana CD, Fidler IJ: Elevated expression of phosphatidylserine in the outer membrane leaflet of human tumor cells and recognition by activated human blood monocytes. Cancer Res 1991, 51:3062-3066.

18. Ran S, Downes A, Thorpe PE: Increased exposure of anionic phospholipids on the surface of tumor blood vessels. Cancer Res 2002, 62:6132-6140.

19. Ran S, Thorpe PE: Phosphatidylserine is a marker of tumor vasculature and a potential target for cancer imaging and therapy. Int J Radiat Oncol Biol Phys 2002, 54:1479-1484

20. Sparreboom A, van Tellingen O, Nooijen WJ, Beijnen JH: Preclinical pharmacokinetics of paclitaxel and docetaxel. Anticancer Drugs 1998, 9:1-17.

21. Tannock IF, De Wit R, Berry WR, Horti J, Pluzanska A, Chi KN, Oudard S, Théodore C, James ND, Turesson I: Docetaxel plus prednisone or mitoxantrone plus prednisone for advanced prostate cancer. $N$ Engl J Med 2004, 351:1502-1512.

22. Huang $X$, Bennett M, Thorpe PE: A monoclonal antibody that binds anionic phospholipids on tumor blood vessels enhances the antitumor effect of docetaxel on human breast tumors in mice. Cancer Res 2005, 65:4408-4416.

23. Van Rite BD, Lazrak YA, Pagnon ML, Palwai NR, Neves LFF, McFetridge PS, Harrison RG: Enzyme prodrug therapy designed to target I-methioninase to the tumor vasculature. Cancer Lett 2011, 301:177-184

24. Van Rite BD, Harrison RG: Annexin V-targeted enzyme prodrug therapy using cytosine deaminase in combination with 5-fluorocytosine. Cancer Lett 2011, 307:53-61.

25. Krais JJ, De Crescenzo O, Harrison RG: Purine Nucleoside Phosphorylase targeted by Annexin $\mathrm{V}$ to breast cancer vasculature for enzyme prodrug therapy. PLOS ONE 2013, 8:e76403.

26. Esaki N, Tanaka H, Uemura S, Suzuki T, Soda K: Catalytic action of L-methionine .gamma.-lyase on selenomethionine and selenols. Biochemistry (Mosc) 1979, 18:407-410.

27. Hori H, Takabayashi K, Orvis L, Carson DA, Nobori T: Gene cloning and characterization of pseudomonas putida I-Methionine-a-deamino- $\gamma$-mercaptomethane-lyase. Cancer Res 1996, 56:2116-2122.

28. Poirson-Bichat F, Gonfalone G, Bras-Goncalves R, Dutrillaux B, Poupon M: Growth of methionine-dependent human prostate cancer (PC-3) is inhibited by ethionine combined with methionine starvation. Br J Cancer 1997, 75:1605-1612.

29. Zhao R, Domann FE, Zhong W: Apoptosis induced by selenomethionine and methioninase is superoxide mediated and p53 dependent in human prostate cancer cells. Mo Cancer Ther 2006, 5:3275-3284.

30. Plunkett W, Gandhi V, Huang P, Robertson L, Yang L, Gregoire V, Estey E, Keating M: Fludarabine: pharmacokinetics, mechanisms of action, and rationales for combination therapies. Semin Oncol 1993, 20:2-12.
31. Parker WB, Allan PW, Shaddix SC, Rose LM, Speegle HF, Gillespie GY, Bennett LL Jr: Metabolism and metabolic actions of 6-methylpurine and 2fluoroadenine in human cells. Biochem Pharmacol 1998, 55:1673-1681.

32. Sorscher EJ, Peng S, Bebok Z, Allan PW, Bennett LL Jr, Parker WB: Tumor cell bystander killing in colonic carcinoma utilizing the Escherichia coli DeoD gene to generate toxic purines. Gene Ther 1994, 1:233-238.

33. Singh PP, Joshi S, Russell PJ, Verma ND, Wang X, Khatri A: Molecular chemotherapy and chemotherapy: a new front against Late-Stage Hormone-Refractory Prostate Cancer. Clin Cancer Res 2011, 17:4006-4018.

34. Xie X, Guo J, Kong Y, Xie GX, Li L, Lv N, Xiao X, Tang J, Wang X, Liu P: Targeted expression of Escherichia coli purine nucleoside phosphorylase and Fludara ${ }^{\oplus}$ for prostate cancer therapy. J Gene Med 2011, 13:680-691.

35. Voeks D, Martiniello-Wilks R, Madden V, Smith K, Bennetts E, Both GW, Russell PJ: Gene therapy for prostate cancer delivered by ovine adenovirus and mediated by purine nucleoside phosphorylase and fludarabine in mouse models. Gene Ther 2002, 9:759-768.

36. Longey D: 5-fluorouracil, mechanisms of action and clinical strategies. Nat Rev Cancer 2003, 3:330-338.

37. Miller CR, Williams CR, Buchsbaum DJ, Gillespie GY: Intratumoral 5-fluorouracil produced by cytosine deaminase/5-fluorocytosine gene therapy is effective for experimental human glioblastomas. Cancer Res 2002, 62:773-780

38. Van Rite BD, Krais JJ, Cherry M, Sikavitsas VI, Kurkjian C, Harrison RG: Antitumor activity of an enzyme prodrug therapy targeted to the breast tumor vasculature. Cancer Invest 2013, 31:505-510.

39. Zang XP, Palwai NR, Lerner MR, Brackett DJ, Pento JT, Harrison RG Targeting a methioninase-containing fusion protein to breast cancer urokinase receptors inhibits growth and migration. Anticancer Res 2006, 26:1745-1751.

40. O'Brien J, Wilson I, Orton T, Pognan F: Investigation of the Alamar Blue (resazurin) fluorescent dye for the assessment of mammalian cell cytotoxicity. Eur J Biochem 2000, 267:5421-5426.

41. Cao S, Durrani FA, Rustum YM: Selective modulation of the therapeutic efficacy of anticancer drugs by selenium containing compounds against human tumor xenografts. Clin Cancer Res 2004, 10:2561-2569.

42. Yang $Y$, Huang F, Ren $Y$, Xing L, Wu Y, Li Z, Pan H, Xu C: The anticancer effects of sodium selenite and selenomethionine on human colorectal carcinoma cell lines in nude mice. Oncol Res Featur Preclin Clin Cancer Ther 2009, 18:1-8.

43. Van Heerde W, Poort S, Van't Veer C, Reutelingsperger C, De Groot PG: Binding of recombinant annexin $V$ to endothelial cells: effect of annexin $\mathrm{V}$ binding on endothelial-cell-mediated thrombin formation. Biochem J 1994, 302:305-312.

44. Van Heerde WL, Sakariassen KS, Hemker HC, Sixma JJ, Reutelingsperger C, de Groot PG: Annexin $V$ inhibits the procoagulant activity of matrices of TNF-stimulated endothelium under blood flow conditions. Arterioscler Thromb Vasc Biol 1994, 14:824-830.

45. Afshar S, Asai T, Morrison SL: Humanized ADEPT comprised of an engineered human purine nucleoside phosphorylase and a tumor targeting peptide for treatment of cancer. Mol Cancer Ther 2009, 8:185-193.

46. Stone E, Paley O, Hu J, Ekerdt B, Cheung N-K, Georgiou G: De novo engineering of a human cystathionine- $\gamma$-lyase for systemic I-Methionine depletion cancer therapy. ACS Chem Biol 2012, 7:1822-1829.

47. Yang Z, Wang J, Lu Q, Xu J, Kobayashi Y, Takakura T, Takimoto A, Yoshioka T, Lian C, Chen C: PEGylation confers greatly extended half-life and attenuated immunogenicity to recombinant methioninase in primates. Cancer Res 2004 , 64:6673-6678.

doi:10.1186/s12929-014-0065-3

Cite this article as: Guillen et al:: Targeted enzyme prodrug therapy for metastatic prostate cancer - a comparative study of L-methioninase, purine nucleoside phosphorylase, and cytosine deaminase. Journal of Biomedical Science 2014 21:65. 